\title{
Mitochondrial Haplotype Analysis for Differentiation of Isolates of Phytophthora cinnamomi
}

\author{
F. N. Martin and M. D. Coffey
}

First author: United States Department of Agriculture-Agricultural Research Service, 1636 East Alisal St., Salinas, CA 93905; and second author: Plant Pathology and Microbiology, University of California, Riverside 92521.

Accepted for publication 29 September 2011.

\section{ABSTRACT}

Martin, F. N., and Coffey, M. D. 2012. Mitochondrial haplotype analysis for differentiation of isolates of Phytophthora cinnamomi. Phytopathology 102:229-239.

Although Phytophthora cinnamomi is heterothallic, there are few instances of successful crossing in laboratory experiments, and analysis of field populations indicates a clonally reproducing population. In the absence of sexual recombination, the ability to monitor mitochondrial haplotypes may provide an additional tool for identification of clonal isolates and analysis of population structure. To determine mitochondrial haplotypes for this species, seven mitochondrial loci spanning a total of $6,961 \mathrm{bp}$ were sequenced for 62 isolates representing a geographically diverse collection of isolates with A1 and A2 mating type. Three of the regions were primarily intergenic regions between $\operatorname{trn} G$ and $r n s$, $r n s$ and nad3, and nad6 and coxl, while the remaining loci spanned cox2, nad9, rps10, and $\sec Y$ coding regions and some of the flanking spacer regions. In total, 45 mitochondrial haplotypes were identified (75\% of the total isolates examined) with differences due to single-nucleotide polymorphisms (SNPs, totaling $152 \mathrm{bp}$ ) and length mutations (17 indels $>2 \mathrm{bp}$ representing a total of $910 \mathrm{bp}$ in length). SNPs were the predominate mutation in the four coding regions and their flanking intergenic regions, while both SNPs and length mutations were observed in the three primarily intergenic regions. Some of the length mutations in these regions were due to addition or loss of unique sequences while others were due to variable numbers of subrepeats (in the trnG-rns region, there were 3 to 12 copies of a 24-bp subrepeat sequence that differentiated 17 haplotypes). Network analysis of the haplotypes identified eight primary clades, with the most divergent clade representing primarily A1 isolates collected from Papua New Guinea. The isolate grouping in the network corresponded to mating type and previously published isozyme classifications, with three exceptions: a haplotype representing an A1 mating type (H29) was placed well within the A2 mating type haplotype grouping, one haplotype (H26) had isolates with two isozyme classifications, and one isozyme group was represented on separate network clades, suggesting that recombination has occurred in the past. Among the 62 isolates examined, several examples were identified of isolates recovered from different geographic regions having the same mitochondrial haplotype, suggesting movement of isolates via plant material. Analysis of the data set to determine whether fewer loci could be sequenced to classify haplotypes indicated that the trnG-rns and rns-nad6 loci would classify $87 \%$ of the haplotypes identified in this study, while additional sequencing of the nad 9 or $\sec Y$ loci would further differentiate the remaining six haplotypes. Based on conservation of gene order in Phytophthora spp., the trnG-rns locus should be useful for mitochondrial haplotype classification in other species, as should the $\operatorname{cox} 2, \operatorname{nad} 9, \operatorname{rps} 10$, and $\sec Y$ loci. However, the $r n s$ nad3 and nad6-cox1 loci span regions that can have a different gene order in some Phytophthora spp.

Additional keywords: haplotype network.
Phytophthora cinnamomi was initially described by Rands as the cause of a canker on Cinnamomum burmannii in Burma in 1922 (40) and has since been observed to have a worldwide distribution with a broad host range encompassing a wide range of horticultural crops and native forest vegetation. This species is best known for its devastating impact on native forest ecosystems in Australia, where it has caused what is commonly referred to as Jarrah dieback (4). In the southwestern region of Australia alone, there are $>2,285$ species of susceptible plants (43), which adds to the difficulty of managing this pathogen. The center of origin for $P$. cinnamomi is believed to be in the Papua New Guinea area due

\section{Corresponding author: F. N. Martin; E-mail address: frank.martin@ars.usda.gov}

Mention of trade names or commercial products in this manuscript is solely for the purpose of providing specific information and does not imply recommendation or endorsement by the United States Department of Agriculture. The United States Department of Agriculture is an equal opportunity provider and employer.

* The $\boldsymbol{e}$-Xtra logo stands for "electronic extra" and indicates that the online version contains a supplementary file.

http://dx.doi.org/10.1094/PHYTO-04-11-0115

This article is in the public domain and not copyrightable. It may be freely reprinted with customary crediting of the source. The American Phytopathological Society, 2012 to the high level of genotypic diversity among isolates from this location observed in isozyme (35) as well as random amplified polymorphic DNA (RAPD) and restriction fragment length polymorphism (RFLP) studies (22).

Although $P$. cinnamomi is a heterothallic species, it can be difficult to get oospores to form when pairing isolates of opposite mating type and, when oospores do form, they can be difficult to germinate (16-18,32,44). However, Linde et al. (23) and Dobrowolski et al. (7) were successful in obtaining oospores from sexual outcrosses with sufficient germination frequency to allow analysis of the progeny. The ability of isolates to self and form oospores in single culture in response to different environmental stimuli has been reported but, again, the oospores are difficult to germinate $(32,41,48)$. Interestingly, Jayasekera et al. (16) observed selfing of isolates in colonized roots of Lupinus angustifolis when placed in soil underneath Acacia pulchella. The occurrence of sexual recombination in populations of $P$. cinnamomi is important because this could assist with long-term survival of the pathogen in the form of persistent oospores as well as increasing the potential for genetic diversity of the pathogen.

Isozyme analysis has been useful for identification of intraspecific variation in a range of Phytophthora spp. (10), including $P$. cinnamomi. Old et al. $(34,35)$ examined a collection of isolates from Australia and observed low levels of genetic diversity and 
heterozygosity, concluding that the population was not sexually recombining. Linde et al. (21) reported the same conclusion for a population from South Africa; although 9 multilocus genotypes were identified, for 14 of these isozyme loci, there were low levels of heterozygosity and genotypic diversity. Oudemans and Coffey (36) examined a worldwide collection of isolates and identified eight multilocus isozyme genotypes and, like Old et al. $(34,35)$ and Linde et al. (21), found clear separation of A1 and A2 mating type isolates.

A more detailed analysis of population structure has been done with several different molecular markers. Linde et al. (22) used RAPD and RFLP analysis with their South African population and reported the same results they observed with isozyme analysis: the population was clonally reproducing. Interestingly, isolates from the Australian population were also included in the analysis and found to be almost identical to the South African population, leading the authors to conclude that both populations were introduced from the same location. The Australian population of this pathogen was examined in greater detail by Dobrowolski et al. $(6,8)$ using microsatellite analysis and found to consist primarily of three clonal lineages (one for A1 mating type isolates and two for A2) that corresponded with the isozyme groupings of Old et al. $(34,35)$, further supporting the conclusion that the pathogen was introduced. More recently, populations recovered from ornamental crops in the southeastern United States were analyzed using amplified fragment length polymorphism (AFLP) analysis (9). Isolates clustered in four primary clades in this analysis, with the majority of isolates in a single clade and only two of the isolates examined having an A1 mating type.

When working with predominantly clonally reproducing populations in which sexual recombination is either exceedingly rare or does not occur, the mitochondrial genome may provide an additional marker for analyzing populations. A good example for how useful mitochondrial haplotype analysis can be in Phytophthora spp. is the procedure initially described by Carter et al. (5) for $P$. infestans that is now widely used in population studies of this species. Because the mitochondrial genome is uniparentally inherited from the maternal parent in sexual outcrosses $(11,47)$, in the absence of sexual recombination, the mitochondrial haplotype should be a good marker for following clonally reproducing populations. Early work using the mitochondrial genome as a marker focused on RFLP analysis for identification of species and, in some cases, subgroupings of a species (10). More recently, polymerase chain reaction (PCR)-RFLP analysis of the coxl and 2 gene cluster was found to be useful for identification of species, although intraspecific variation of $P$. cinnamomi was not identified in the four isolates examined (30). Sequence analysis of mitochondrial genes ( $\operatorname{cox} 1, \operatorname{cox} 2$, and nadl) has been used for phylogenetic analysis of the genus and, although useful for classification or identification of species, the levels of intraspecific variation tend to be low $(19,28,29,46)$. In contrast, intergenic regions of the mitochondrial genome of Phytophthora spp. have been a useful source of inter- and intraspecific polymorphisms $(24,31,42,45)$.

The availability of whole mitochondrial genome data for $P$. infestans $(1,37)$, P. ramorum, P. sojae $(26,27)$, and 17 other Phytophthora spp. (including P. cinnamomi) and 14 Pythium spp. in an ongoing mitochondrial genome sequencing project (F. Martin, unpublished) provided an opportunity to use comparative genomic approaches to identify regions of the genome that might be useful for inter- and intraspecific analysis of Phytophthora spp. The ability to do alignments across genera allowed for selection of specific loci useful for phylogenetic analysis and the development of highly conserved primers for their amplification. Likewise, the ability to examine the mitochondrial genome of $P$. cinnamomi and compare gene order differences with other species in the genus Phytophthora identified intergenic regions that might be more prone to intraspecific variation. The objective of this experimentation was to evaluate these mitochondrial loci at a DNA sequence level for their ability to differentiate mitochondrial haplotypes of $P$. cinnamomi in a geographically diverse collection of isolates.

\section{MATERIALS AND METHODS}

Cultures used. The 62 isolates used in this investigation were from the World Phytophthora Genetic Resource Collection at the University of California, Riverside (Table 1). Cultures were grown and DNA extracted as previously described (3).

Template amplification and sequencing. Loci to include in the analysis were identified by a comparative genomics approach using complete mitochondrial genome sequences from a range of Pythium and Phytophthora spp. (F. Martin, unpublished). Four of the loci were coding regions and their flanking spacer sequences (cox2, nad9, rps10, and ymf16) that have been used for phylogenetic analysis of Phytophthora spp. (26) (F. N. Martin, unpublished). Three loci (trnG-rns, rns-nad3, and nad6-coxl) were spacer regions that varied in size among the mitochondrial genomes of Phytophthora spp. that had been sequenced. DNA sequences for the flanking coding regions were extracted from the genomic sequences and conserved regions used to design amplification primers (Table 2). All amplifications were done using $\approx 10 \mathrm{ng}$ of template DNA, $0.5 \mathrm{mM}$ forward and reverse primers, 2 or $3 \mathrm{mM} \mathrm{MgCl}_{2}$ (Table 2), $100 \mu \mathrm{M}$ dNTP, $1 \times$ amplification buffer, and 1 unit of AmpliTaq (Applied Biosystems, Foster City, $\mathrm{CA}$ ) in a volume of $25 \mu \mathrm{l}$. Templates were amplified in an ABI 9600 thermal cycler with the following cycling conditions: 1 interval of $95^{\circ} \mathrm{C}$ for $3 \mathrm{~min} ; 35$ cycles of $95^{\circ} \mathrm{C}$ for $1 \mathrm{~min}, 1 \mathrm{~min}$ of annealing for the indicated temperature (Table 2), and extension at $72^{\circ} \mathrm{C}$ for $2 \mathrm{~min}$; and 1 interval of $72^{\circ} \mathrm{C}$ for $5 \mathrm{~min}$ followed by a $4^{\circ} \mathrm{C}$ hold. After confirming template amplification by running samples on an agarose gel, sequencing templates were prepared by treatment with ExoSap (USB, Cleveland, $\mathrm{OH}$ ) in accordance with the manufacturer's instructions and sent to the Nucleic Acid Sequencing Facility at the Penn State University (University Park, PA) for sequencing with the amplification primers unless otherwise noted (Table 2). Each template was sequenced in both directions to generate a consensus sequence based on complementary strands. Sequencher 4.7 (Gene Codes, Ann Arbor, MI) was used to generate consensus sequences while DS Gene v. 2.5 (Accelrys, San Diego, CA) was used for making alignments.

Haplotype analysis. Sequences for each region were aligned by Clustal W in DS Gene v2.5, with McClade v. 4.02 (Sinaur Associates, Sunderland, MA) used to fine tune the alignment. Polymorphisms in the alignments among the isolates for each individual region were noted and classified as a separate haplotype; examples for each haplotype have been deposited in GenBank (Table 3). Once the analysis of the individual loci was completed, the results for all seven loci were combined and a numeral designation for each final haplotype was given (Table 1). To confirm the final haplotype classification, the individual alignments were concatenated in to a single alignment file and analyzed using DnaSP 5.10.01 (20). To visualize in a cladogram the relationships among the different mitochondrial haplotypes based on the total number of base differences, the concatenated sequence alignment was modified to ensure that indels were accounted for by inserting standard IUPAC abbreviation codes in gaps corresponding to bases that were not present in the alignment. Unweighted pairgroup with arithmetic means analysis of the modified alignment was run in PAUP v. $4.0 \mathrm{~b} 10$ (Sinaur Associates).

A haplotype network visualizing the relationship among the various haplotypes was calculated in SplitsTree v. 4.1 (14) using the unmodified data set (IUPAC codes were not inserted) with uncorrected $\mathrm{P}$ and a NeighborNet network calculation. To reduce 
the complexity of the network, a neighbor-joining network was constructed and 1,000 replicates of bootstrap analysis were run to determine statistical support for the branching. Network 4.5.1.6 (Fluxus Technology Ltd., Clare, Suffolk, England) was also used to confirm the network topography and overall grouping of haplotypes using maximum parsimony (MP). A median joining network calculation (2) was completed using the default 1:1 transition/transversion ratio and $\varepsilon=0$. When the data set was examined in PAUP v. $4.0 \mathrm{~b} 10$ to determine the transition/transversions ratio, it was found to be 1.57 ; changing this value to $1: 2$

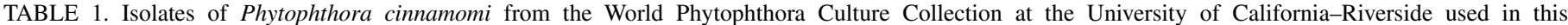

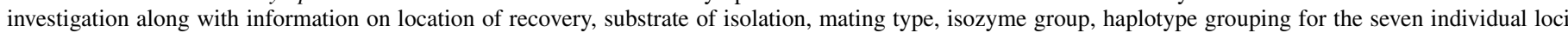
sequenced, and final mitochondrial haplotype grouping

\begin{tabular}{|c|c|c|c|c|c|c|c|c|c|c|c|c|}
\hline $\begin{array}{l}\text { Accession } \\
\text { number }\end{array}$ & Host & Location & $\begin{array}{l}\text { Mating } \\
\text { type }\end{array}$ & Isozyme $^{\mathrm{a}}$ & $\operatorname{cox} 2$ & $\operatorname{nad} 9$ & rps10 & $\sec Y$ & $\begin{array}{c}\text { trnG- } \\
r n s\end{array}$ & $\begin{array}{l}\text { nad6- } \\
\operatorname{cox} 1\end{array}$ & $\begin{array}{l}\text { rns- } \\
\text { nad3 }\end{array}$ & $\begin{array}{c}\text { Mitochondrial } \\
\text { haplotype }\end{array}$ \\
\hline P10162 & Persea americana & Puerto Rico & A2 & - & 1 & 4 & 1 & 4 & 19 & 6 & 17 & 1 \\
\hline P11307 & Rhododendron sp. & Poland & $\mathrm{A} 2$ & - & 1 & 4 & 1 & 4 & 16 & 6 & 17 & 2 \\
\hline P15348 & P. americana & Spain $^{\mathrm{b}}$ & $\mathrm{A} 2$ & - & 1 & 4 & 1 & 4 & 11 & 6 & 17 & 3 \\
\hline P2425 & P. americana & South Africa & $\mathrm{A} 2$ & - & 1 & 4 & 1 & 4 & 11 & 6 & 17 & 3 \\
\hline P15887 & Quercus suber & Portugal & $\mathrm{A} 2$ & - & 1 & 4 & 1 & 4 & 16 & 6 & 18 & 4 \\
\hline P6493 & Rhododendron sp. & China & A1 & $\operatorname{cinn} 8$ & 1 & 10 & 4 & 1 & 9 & 5 & 16 & 5 \\
\hline P3237 & Camellia sp. & China & A1 & $\operatorname{cinn} 1$ & 1 & 17 & 1 & 2 & 10 & 3 & 10 & 6 \\
\hline P6492 & Camellia sp. & China & A1 & $\operatorname{cinn} 1$ & 1 & 17 & 1 & 2 & 10 & 3 & 10 & 6 \\
\hline P2284 & Erica sp. & Switzerland & A2 & $\operatorname{cinn} 4$ & 1 & 14 & 2 & 1 & 8 & 2 & 19 & 7 \\
\hline P2301 & Rhododendron sp. & California & $\mathrm{A} 2$ & cinn5 & 1 & 14 & 2 & 1 & 8 & 2 & 20 & 8 \\
\hline P11312 & Pinus mиgo & Poland & $\mathrm{A} 2$ & - & 1 & 1 & 2 & 1 & 8 & 1 & 23 & 9 \\
\hline P11320 & Quercus sp. & Poland & A2 & - & 1 & 1 & 2 & 1 & 8 & 1 & 23 & 9 \\
\hline P15883 & Q. suber & Spain & A2 & - & 1 & 1 & 2 & 1 & 8 & 1 & 23 & 9 \\
\hline P2400 & Persea americana & Hawaii & A2 & $\operatorname{cinn} 4$ & 1 & 1 & 2 & 1 & 8 & 1 & 23 & 9 \\
\hline P2428 & P. americana & California & $\mathrm{A} 2$ & $\operatorname{cinn} 4$ & 1 & 1 & 2 & 1 & 8 & 1 & 23 & 9 \\
\hline P3665 & Eucalyptus sp. & Australia & $\mathrm{A} 2$ & $\operatorname{cinn} 4$ & 1 & 1 & 2 & 1 & 8 & 1 & 23 & 9 \\
\hline P15347 & P. americana & Spain $^{c}$ & $\mathrm{~A} 2$ & - & 1 & 3 & 2 & 1 & 8 & 1 & 23 & 10 \\
\hline P2288 & Pinus radiata & California & $\mathrm{A} 2$ & $\operatorname{cinn} 4$ & 1 & 1 & 2 & 1 & 9 & 1 & 23 & 11 \\
\hline $\mathrm{P} 2475$ & Persea americana & California & A2 & $\operatorname{cinn} 4$ & 1 & 1 & 2 & 1 & 9 & 1 & 23 & 11 \\
\hline $\mathrm{P} 2110(\mathrm{~T})^{\mathrm{c}}$ & Cinnamomum burmannii & West Sumatra & A2 & $\operatorname{cinn} 4$ & 1 & 1 & 2 & 1 & 7 & 1 & 23 & 12 \\
\hline P6304 & P. americana & North Sulawesi & A2 & $\operatorname{cinn} 4$ & 1 & 1 & 2 & 1 & 7 & 1 & 23 & 12 \\
\hline P6305 & P. americana & Indonesia & $\mathrm{A} 2$ & $\operatorname{cinn} 4$ & 1 & 1 & 2 & 1 & 7 & 1 & 23 & 12 \\
\hline P2424 & Rhododendron sp. & California & A2 & - & 1 & 14 & 2 & 1 & 7 & 2 & 19 & 13 \\
\hline P3232 & Soil (Rhododendron sp.) & China & A2 & $\operatorname{cinn} 5$ & 1 & 15 & 2 & 1 & 7 & 2 & 21 & 14 \\
\hline P15349 & P. americana & Spain & A2 & - & 1 & 1 & 2 & 1 & 14 & 1 & 24 & 15 \\
\hline P11596 & Arbutus menziesii & California & A2 & - & 1 & 1 & 2 & 1 & 14 & 1 & 23 & 16 \\
\hline P11600 & A. menziesii & California & A2 & - & 1 & 1 & 2 & 1 & 14 & 1 & 23 & 16 \\
\hline P15378 & P. americana & Spain & $\mathrm{A} 2$ & - & 1 & 1 & 2 & 1 & 13 & 1 & 23 & 17 \\
\hline P15881 & Q. suber & Portugal & A2 & - & 1 & 1 & 2 & 1 & 13 & 1 & 23 & 17 \\
\hline P10203 & Camellia sp. & South Carolina & A1 & - & 1 & 16 & 1 & 2 & 12 & 3 & 11 & 18 \\
\hline P2138 & P. americana & California & A1 & cinn 1 & 1 & 16 & 1 & 2 & 12 & 3 & 9 & 19 \\
\hline P2021 & Camellia sp. & California & $\mathrm{A} 1$ & cinn 1 & 1 & 16 & 1 & 2 & 12 & 3 & 10 & 20 \\
\hline P2096 & Camellia japonica & California & A1 & cinn 1 & 1 & 16 & 1 & 2 & 12 & 3 & 10 & 20 \\
\hline P15821 & Soil & Papua New Guinea & A1 & - & 1 & 16 & 1 & 2 & 21 & 3 & 10 & 21 \\
\hline P11558 & Camellia sp. & Germany & A1 & - & 1 & 16 & 1 & 2 & 18 & 3 & 9 & 22 \\
\hline P2100 & C. japonica & California & A1 & cinn 1 & 1 & 16 & 1 & 2 & 18 & 3 & 10 & 23 \\
\hline P2399 & Camellia sp. & California & A1 & $\operatorname{cinn} 1$ & 1 & 16 & 1 & 2 & 18 & 3 & 10 & 23 \\
\hline P15822 & Soil & Papua New Guinea & $\mathrm{A} 2$ & - & 1 & 1 & 2 & 1 & 20 & 1 & 23 & 24 \\
\hline P2370 & Vitus sp. & South Africa & A1 & $\operatorname{cinn} 3$ & 10 & 18 & 1 & 3 & 15 & 15 & 12 & 25 \\
\hline P10140 & Vitus sp. & South Africa & A1 & - & 10 & 18 & 1 & 3 & 28 & 15 & 12 & 26 \\
\hline P2121 & P. americana & Madagascar & A1 & $\operatorname{cinn} 2$ & 10 & 18 & 1 & 3 & 28 & 15 & 12 & 26 \\
\hline P2371 & Leuc. argenteum & South Africa & A1 & $\operatorname{cinn} 3$ & 10 & 18 & 1 & 3 & 28 & 15 & 12 & 26 \\
\hline P2159 & Vitus sp. & South Africa & A1 & $\operatorname{cinn} 3$ & 10 & 18 & 1 & 5 & 28 & 15 & 12 & 27 \\
\hline P2160 & Vitus sp. & South Africa & A1 & - & 10 & 18 & 1 & 3 & 22 & 15 & 12 & 28 \\
\hline P10781 & Eustoma grandiflorum & Japan & A1 & - & 1 & 2 & 2 & 1 & 3 & 2 & 22 & 29 \\
\hline P15839 & Hypericum sp. & Taiwan & A1 & - & 1 & 2 & 2 & 1 & 3 & 2 & 22 & 29 \\
\hline P15314 & P. americana & Spain & A2 & - & 1 & 1 & 2 & 1 & 2 & 1 & 23 & 30 \\
\hline P15332 & P. americana & Spain & A2 & - & 1 & 1 & 2 & 11 & 5 & 1 & 23 & 31 \\
\hline P6379 & Annus comosus & Taiwan & A1 & cinn 1 & 9 & 16 & 1 & 2 & 6 & 3 & 9 & 32 \\
\hline P15838 & Cinnamomomum kotoense & Taiwan & A1 & - & 1 & 11 & 5 & 1 & 5 & 8 & 15 & 33 \\
\hline P6490 & P. americana & Florida & A2 & $\operatorname{cinn} 4$ & 1 & 1 & 2 & 1 & 1 & 1 & 23 & 34 \\
\hline P15837 & Lilium sp. & Netherlands & A1 & - & 7 & 13 & 1 & 1 & 4 & 4 & 14 & 35 \\
\hline P10933 & Forest soil & China & A1 & - & 2 & 12 & 3 & 9 & 23 & 7 & 13 & 36 \\
\hline P15824 & Soil & Papua New Guinea & A1 & - & 7 & 9 & 1 & 10 & 29 & 9 & 8 & 37 \\
\hline P3662 & C. araucaria & Papua New Guinea & A1 & cinn6 & 1 & 5 & 1 & 6 & 27 & 14 & 7 & 38 \\
\hline P3660 & Forest & Papua New Guinea & A1 & $\operatorname{cinn} 6$ & 5 & 6 & 1 & 6 & 26 & 13 & 4 & 39 \\
\hline P3664 & Eucalyptus sp. & Australia & A1 & $\operatorname{cinn} 2$ & 10 & 18 & 1 & 3 & 26 & 13 & 4 & 40 \\
\hline P3657 & Forest & Papua New Guinea & A1 & cinn6 & 1 & 6 & 1 & 6 & 26 & 11 & 5 & 41 \\
\hline P3656 & Forest & Papua New Guinea & A1 & $\operatorname{cinn} 7$ & 6 & 4 & 1 & 6 & 24 & 10 & 6 & 42 \\
\hline P2183 & Soil (Rhododendron sp.) & Papua New Guinea & A1 & - & 4 & 6 & 1 & 6 & 17 & 9 & 2 & 43 \\
\hline P3659 & Araucaria forest & Papua New Guinea & A1 & cinn6 & 8 & 7 & 1 & 8 & 25 & 12 & 3 & 44 \\
\hline P3658 & C. araucaria & Papua New Guinea & A1 & cinn6 & 3 & 8 & 1 & 7 & 30 & 9 & 1 & 45 \\
\hline
\end{tabular}

a Isozyme determined in Oudemans and Coffey (36); $-=$ not determined.

b Isolate recovered from plant material imported from South Africa.

c $\mathrm{T}=$ type culture. 
in Network 4.5.1.6 and rerunning the analysis had no meaningful effect on the results. Likewise, higher values of $\varepsilon$ were evaluated and, although the haplotype groupings remained the same, the hubs interconnecting the different groupings became much more complex, with many alternative pathways that complicated interpretation of the results. After the first median joining network calculation was run, the data set underwent MP post processing to delete links not used by the shortest trees in the network (38) and the network was redrawn.

Phylogenetic analysis. A partition homogeneity test of the concatenated unmodified data set was run in PAUP v. $4.0 \mathrm{~b} 10$ and phylogenetic relationships were evaluated among isolates by several methods. PAUP v. $4.0 \mathrm{~b} 10$ was used to conduct MP analysis with a heuristic tree search with MULPARS on, steepest decent

TABLE 2. Primers used for amplification and sequencing in this investigation

\begin{tabular}{|c|c|c|c|c|}
\hline Marker & Amplification primers & $\begin{array}{l}\text { Amplicon } \\
\text { size (bp) }\end{array}$ & $\begin{array}{c}\mathrm{MgCl}_{2} \\
\text { concentration }\end{array}$ & $\begin{array}{c}\text { Annealing } \\
\text { temperature }\left({ }^{\circ} \mathrm{C}\right)\end{array}$ \\
\hline \multirow[t]{2}{*}{$\operatorname{cox} 2+$ spacer $^{b}$} & FM 35 CAGAACCTTGGCAATTAGG & $\ldots$ & $\ldots$ & $\ldots$ \\
\hline & Phy10b GCAAAAGCACTAAAAATTAAATATAA & 1,041 & 3 & 54 \\
\hline \multirow[t]{2}{*}{ nad9 } & Nad9- F TACAACAAGAATTAATGAGAAC & $\ldots$ & $\ldots$ & $\ldots$ \\
\hline & Nad9-R GTTAAAATTTGTACTACTAACAT & 1,024 & 3 & 61 \\
\hline \multirow[t]{2}{*}{ rps10 } & Prv9-F GTATACTCTAACCAACTGAGT & $\ldots$ & $\ldots$ & $\ldots$ \\
\hline & Prv9-R GTTGGTTAGAGTAAAAGACT & 601 & 3 & 59 \\
\hline \multirow{2}{*}{$y m f 16^{\mathrm{b}}$} & SecY-F TCTATCGTGTTTACCAATTTC & $\ldots$ & $\ldots$ & $\ldots$ \\
\hline & SecY-R TAACAAATGGATCTTCTTTAAAA & 946 & 3 & 61 \\
\hline \multirow[t]{2}{*}{$\operatorname{trn} G-r n s$} & Phy Intra-F1 GGTAGAGTATAACCTTGC & $\ldots$ & $\ldots$ & $\ldots$ \\
\hline & Phy Intra-R1 ATAGCATTTATTCTGAGCCA & 1,172 & 2 & 57 \\
\hline \multirow[t]{2}{*}{ rns-nad3 } & RN-CoxF GATGAAGTCGTAACAAGGTA & $\ldots$ & $\ldots$ & $\ldots$ \\
\hline & RN-nad3Rb TGTTTATATTTGGTGTAGTGAT & 1,814 & 2 & 66 \\
\hline \multirow[t]{2}{*}{ nad6-cox 1} & ATP-Nad-R1 TTCAAGAAATTCATTAAAAGCT & $\ldots$ & $\ldots$ & $\ldots$ \\
\hline & Nad-cox1 R1 CTATGAGTGGATGGAATGCT & 673 & 2 & 62 \\
\hline \multicolumn{5}{|c|}{ Sequencing primers } \\
\hline \multirow[t]{2}{*}{ Cox $2+$ spacer } & FM82 TTGGCAATTAGGTTTTCAAGATCC & $\ldots$ & $\ldots$ & $\ldots$ \\
\hline & FM80 AATATCTTTATGATTTGTTGAAA & $\ldots$ & $\ldots$ & $\ldots$ \\
\hline \multirow[t]{3}{*}{ rns-nad3 } & rnsnad F2 TCGTAACAAGGTAGTCGTA & $\ldots$ & $\ldots$ & $\ldots$ \\
\hline & rnsnad Fa CATTTGATATATTCTCAAATGTC & $\ldots$ & $\ldots$ & $\ldots$ \\
\hline & rnsnad Ra TTCTCAAATGTCTCTAAAAAATT & $\ldots$ & $\ldots$ & $\ldots$ \\
\hline nad6-cox 1 & nadcox R3 TGTATATGTAACTTTAATACATG & ... & $\ldots$ & $\ldots$ \\
\hline
\end{tabular}

a Size may vary depending on the isolate, especially for trnG-rns and rns-nad3.

${ }^{\mathrm{b}}$ FM35 was previously reported in Martin (25), FM50 in Martin and Tooley (28), Phy10b in Martin et al. (31), and ymf16 in Martin (26).

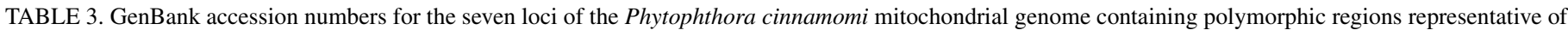
the different mitochondrial haplotype for each locus individually ${ }^{\mathrm{a}}$

\begin{tabular}{|c|c|c|c|c|c|c|c|}
\hline \multirow[b]{2}{*}{ Haplotype } & \multicolumn{7}{|c|}{ Locus } \\
\hline & $\operatorname{cox} 2$ & $\operatorname{nad} 9$ & rps 10 & $\sec Y$ & trnG-rns & rns-nad3 & nad6-cox 1 \\
\hline 1 & JF771366 & JF771741 & JF770958 & JF770595 & JF746380 & JF746309 & JF746209 \\
\hline 2 & JF771369 & JF771748 & JF770972 & JF770640 & JF746332 & JF746293 & JF746219 \\
\hline 3 & JF771354 & JF771739 & JF770961 & JF770613 & JF746343 & JF746310 & JF746255 \\
\hline 4 & JF771340 & JF771727 & JF771018 & JF770581 & JF746341 & JF746311 & JF746217 \\
\hline 5 & JF771356 & JF771779 & JF770978 & JF770612 & JF746342 & JF746308 & JF746258 \\
\hline 6 & JF771352 & JF771778 & $\ldots$ & JF770632 & JF746379 & JF746307 & JF746222 \\
\hline 7 & JF771384 & JF771777 & $\ldots$ & JF770630 & JF746367 & JF746312 & JF746201 \\
\hline 8 & JF771355 & JF771776 & $\ldots$ & JF770631 & JF746334 & JF746278 & JF746218 \\
\hline 9 & JF771361 & JF771745 & $\ldots$ & JF770584 & JF746382 & JF746317 & JF746231 \\
\hline 10 & GU221966 & JF771789 & $\ldots$ & JF770599 & JF746381 & JF746319 & JF746245 \\
\hline 11 & $\ldots$ & JF771747 & $\ldots$ & JF770592 & JF746364 & JF746261 & JF746246 \\
\hline 12 & $\ldots$ & JF771730 & $\ldots$ & $\ldots$ & JF746323 & JF746292 & JF746248 \\
\hline 13 & $\ldots$ & JF771746 & $\ldots$ & $\ldots$ & JF746337 & JF746263 & JF746249 \\
\hline 14 & $\ldots$ & JF771768 & $\ldots$ & $\ldots$ & JF746336 & JF746279 & JF746250 \\
\hline 15 & $\ldots$ & JF771772 & $\ldots$ & $\ldots$ & JF746359 & JF746280 & JF746230 \\
\hline 16 & $\ldots$ & JF771786 & $\ldots$ & $\ldots$ & JF746346 & JF746320 & $\ldots$ \\
\hline 17 & $\ldots$ & JF771788 & $\ldots$ & $\ldots$ & JF746355 & JF746260 & $\ldots$ \\
\hline 18 & $\ldots$ & JF771759 & $\ldots$ & $\ldots$ & JF746361 & JF746284 & $\ldots$ \\
\hline 19 & $\ldots$ & $\ldots$ & $\ldots$ & $\ldots$ & JF746322 & JF746301 & $\ldots$ \\
\hline 20 & $\ldots$ & $\ldots$ & $\ldots$ & $\ldots$ & JF746339 & JF746296 & $\ldots$ \\
\hline 21 & $\ldots$ & $\ldots$ & $\ldots$ & $\ldots$ & JF746338 & JF746305 & $\ldots$ \\
\hline 22 & $\ldots$ & $\ldots$ & $\ldots$ & $\ldots$ & JF746354 & JF746281 & $\ldots$ \\
\hline 23 & $\ldots$ & $\ldots$ & $\ldots$ & $\ldots$ & JF746325 & JF746271 & $\ldots$ \\
\hline 24 & $\ldots$ & $\ldots$ & $\ldots$ & $\ldots$ & JF746369 & JF746274 & $\ldots$ \\
\hline 25 & $\ldots$ & $\ldots$ & $\ldots$ & $\ldots$ & JF746372 & $\ldots$ & $\ldots$ \\
\hline 26 & $\ldots$ & $\ldots$ & $\ldots$ & $\ldots$ & JF746373 & $\ldots$ & $\ldots$ \\
\hline 27 & $\ldots$ & $\ldots$ & $\ldots$ & $\ldots$ & JF746374 & $\ldots$ & $\ldots$ \\
\hline 28 & $\ldots$ & $\ldots$ & $\ldots$ & $\ldots$ & JF746353 & $\ldots$ & $\ldots$ \\
\hline 29 & $\ldots$ & $\ldots$ & $\ldots$ & $\ldots$ & JF746340 & $\ldots$ & $\ldots$ \\
\hline 30 & $\ldots$ & $\ldots$ & $\ldots$ & $\ldots$ & JF746371 & $\ldots$ & $\ldots$ \\
\hline
\end{tabular}

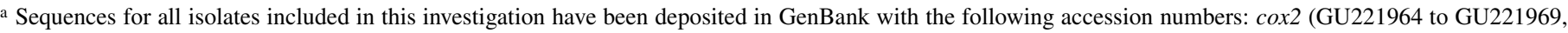
JF771334 to JF771390), nad9 (JF771726 to JF771789), rps10 (JF770957 to JF771018), secY (JF770580 to JF770643), trnG-rns (JF46321 to JF46382), rns-nad3 (JF746259 to JF746320), and nad6-coxl (JF46197 to JF46258). 
option off, random addition of sequences (10 replicates), and TBR branch swapping. To determine support for the various clades of the trees, the analysis was bootstrapped with 100 replicates. In view of the large data set, the maximum rearrangement allowed per replicate was $10^{8}$ to prevent the program from running out of memory. Maximum likelihood (ML) and Bayesian analysis (BA) was done using TOPALi v. 2.5 (33), with JModelTest v. 0.1.1 (39) used to determine the appropriate nucleotide substitution model. Based on the values of the Akaike information criterion, Bayesian information criterion, and decision theory performance-based selection for the concatenated data set, the transition nucleotide substitution model was selected. ML analysis was run in TOPALi using PhyML v. 2.4.5 with 100 bootstrap replicates. BA was run in TOPALi using MrBayes v. 3.1.1; two analyses were run simultaneously for 500,000 generations with a $10 \%$ sampling frequency and burn in of $35 \%$. The runs converged after 500,000 generations with the potential scale reduction factor having a value of 1.0. The concatenated sequence data set is available online as Supplementary material.

\section{RESULTS}

Description of loci and types of mutations observed. In total, approximately $6,961 \mathrm{bp}$ of aligned sequences from seven loci (they amplified in all isolates) were used to determine mitochondrial haplotype. Polymorphisms were caused by singlenucleotide polymorphisms (SNPs) and length mutations (indels), with a total of 152 SNPs and 17 indels $>2$ bp in length.

cox 2 + spacer-The 918 bp from this locus included 684 bp of the $3^{\prime}$ end of the cox 2 gene, 12 bp of spacer, $99 \mathrm{bp}$ of the putative orf32, $115 \mathrm{bp}$ of spacer, and $8 \mathrm{bp}$ of the $5^{\prime}$ end of the cox 1 gene. Thirteen SNPs were identified with no indels for a total of 10 haplotype groupings for this locus.

nad9-The $928 \mathrm{bp}$ from this locus included $558 \mathrm{bp}$ of the nad9 gene, $312 \mathrm{bp}$ of spacer sequences, and $140 \mathrm{bp}$ of the flanking atp 9 and $c o b$ genes, where the amplification primers were located. Twenty SNPs and 1 indel were identified, with a 26-bp indel for a single isolate located in a spacer region consisting of two 13-bp repeats from the region before the indel. In total, 18 haplotype groupings were identified for this locus.

rps 10 - The $556 \mathrm{bp}$ from this locus included $327 \mathrm{bp}$ of the rps10 gene, two full tRNAs (trn $Q$ and trnR), two partial tRNAs where the amplification primers were located (trnI and $t r n F)$, and $38 \mathrm{bp}$ of spacer sequences. Three SNPs and 1 indel were identified, with the 18-bp indel of a sequence repeat located in the rps10 gene for a single isolate. In total, five haplotype groupings were identified for this locus.

$\sec Y$ (ymf16) - The 826 bp from this locus included 744 bp of the sec $Y$ gene and $82 \mathrm{bp}$ of the putative open reading frame (ORF) $y m f 101$. Thirteen SNPs were identified with no indels for a total of 11 haplotype groupings for this locus.

TrnG-rns spacer-The $\approx 994$ to 1,214 bp from this locus included $302 \mathrm{bp}$ of spacer sequence, two tRNAs (trnG-ucc and $\operatorname{trn} Y)$, part of $\operatorname{trn} G(\mathrm{gcc})$ encoding the forward primer, and a putative ORF variable in size among isolates. In total, 12 SNPs and two indels were identified. Both indels were located in a putative ORF leading to sizes of 531 to $750 \mathrm{bp}$. One 3-bp deletion was found in five isolates, with the second indel highly variable among isolates with a 24-bp sequence that was repeated 3 to 12 times. In total, 30 haplotype groupings were identified for this locus, 17 of which were based on variable numbers of the 24-bp repeat.

rns-nad3 spacer-The $\approx 1,415$ to $1,755 \mathrm{bp}$ from this locus (1,852-bp aligned sequence) included $557 \mathrm{bp}$ of spacer sequence, 1 tRNA $(\operatorname{trn} W)$, and a putative OFR that varied in size for some isolates due to indels ( 825 to $1,095 \mathrm{bp}$ ). This locus was the most variable region examined, with 67 SNPs and 12 different indels identified. Eight of the indels represented unique sequences that had been deleted, with four indels consisting of additional copies of 27- and 36-bp repeated sequences (a 0.66- to 1.66-fold increase in length in two specific loci). All but four of the indels were found only in the A1 mating type isolates from Papua New Guinea, as were 24 of the SNPs. In total, 24 haplotype groupings were identified for this locus.

nad6-coxl spacer-The 622-bp from this locus contained primarily spacer regions $(525 \mathrm{bp})$ with one tRNA $(\operatorname{trnR})$ and the coding regions from the flanking genes where the amplification primers were located. Twenty-four SNPs and 1 indel were identified, with the indel consisting of an additional copy of a 23-bp sequence repeat located in the spacer region for two isolates. In total, 15 haplotype groupings were identified for this locus.

Mitochondrial haplotypes. When the sequences for the seven loci were concatenated for the 62 isolates of $P$. cinnamomi examined, 45 mitochondrial haplotypes were identified (Table 1). Some of the differences among haplotypes were minor (one SNP differentiated haplotypes 9, 10, and 11) whereas, in other instances, the differences were larger and resulted in diverse isolates grouping on a separate branches of the cladogram (haplotype 37 to 45) (Fig. 1). Haplotypes in this separate clade were the most divergent and represented A1 mating type isolates primarily from Papua New Guinea. With the exception of the separation of these mitochondrial haplotypes, the remaining isolates did not exhibit further major segregation in their grouping on the cladogram. Likewise, there was limited grouping of isozyme genotype on the cladogram. There were no examples where opposite mating types had the same haplotype but there was one example where a haplotype (H26) had two different isozyme types.

There was some association of mitochondrial haplotype and host, suggesting that distribution on plant material may contribute to geographic spread. An example of this is found with isolates recovered from $P$. americana; $\mathrm{H} 3$ isolates were recovered from this host in Spain and South Africa while H9 isolates were recovered from Hawaii and California (other haplotype 9 isolates were recovered from different tree hosts in Poland and Australia). Likewise, H29 isolates were recovered from horticultural hosts in Japan and Taiwan. Highly similar haplotypes differing by only one or two SNPs were also found on isolates from different regions. For example, $\mathrm{H} 15, \mathrm{H} 16$, and $\mathrm{H} 17$ isolates were recovered from several tree species in Spain, Portugal, and California. This was also observed for isolates recovered from Camelia spp. (H22 and H23 isolates were recovered from Germany and California and $\mathrm{H} 18$ and $\mathrm{H} 20$ isolates were recovered from South Carolina and California) and horticultural crops in Switzerland and California ( $\mathrm{H} 7$ and $\mathrm{H} 8$ ).

Haplotype network and phylogenetic analysis. The neighborjoining haplotype network generated using SplitsTree v. 4.10 generated a network with eight primary branches that were well supported by bootstrap analysis (Fig. 2). The similarities among haplotypes on a specific clade are reflected by their proximity on the network. For example, for the clade with $\mathrm{H} 1$ to $\mathrm{H} 4$, several unique SNPs and two indels (27 and $12 \mathrm{bp}$ in the rns-nad3 region) differentiated this clade from the other haplotypes while different numbers of a 24-bp subrepeat in the trnG-rns region differentiated $\mathrm{H} 3, \mathrm{H} 2$, and $\mathrm{H} 1$ (six, five, and four copies, respectively), with $\mathrm{H} 4$ having five copies and an additional 36-bp insertion in the rns-nad3 region. From reviewing the data for specific clusters of haplotypes on the same clade, varying numbers of the 24-bp subrepeat in the trnG-rns region was often the mutation differentiating these haplotypes. The most divergent clade was the group of A1 isolates recovered from Papua New Guinea; this clade was separated from the other haplotypes by two deletions in the rns-nad3 region (11 and $282 \mathrm{bp}$ ), with variation within the clade caused by isolate-specific SNPs and indels. There was a consistent correlation between haplotype grouping on the neighbor-joining network with mating type and isozyme grouping, with several exceptions; H29 has an A1 mating type but grouped well 
within the A2 mating type haplotypes, $\mathrm{H} 26$ had one isolate each that was in isozyme group 2 and 3, and $\mathrm{H} 7$ was in isozyme group 4 yet it grouped with isozyme group 5 haplotypes H8 and H14.

Rerunning the analysis using MP in Network 4.5.1.6 provided nearly identical results, with several notable exceptions; some of the longer branch lengths were shorter, H5 and H33 were on independent branches (there was poor bootstrap support for the grouping of these haplotypes in Fig. 2), and H36 was terminal to the $\mathrm{H} 1$ to $\mathrm{H} 4$ clade (although $\mathrm{H} 36$ branches off from the $\mathrm{H} 1$ to $\mathrm{H} 4$ clade close to the main backbone of the network in the SplitsTree network in Figure 2, this has poor bootstrap support). Phylogenetic analysis using MP, ML, and BA generated trees that had the same topography and strong bootstrap support for the major branching patterns, as observed with the haplotype network (Fig. 3).

Simplified haplotype determination. Rather than sequence all seven loci to determine mitochondrial haplotype, the data for the individual loci were grouped in different combinations and haplotype analysis was run in DnaSP 5.10.01 in an effort to determine the minimum number of loci needed for accurate classification for the haplotypes identified in this study. Combining data for trnG-rns and rns-nad3 differentiated 39 of the 45 haplotypes, with the remaining 6 haplotypes grouping into three groups of two haplotypes each (H9 and H10, H26 and H27, and H39 and $\mathrm{H} 40$ ). For isolates that fit within $\mathrm{H} 9$ and $\mathrm{H} 10$ or $\mathrm{H} 39$ and $\mathrm{H} 40$, sequencing the nad9 locus would differentiate individual haplo-



Fig. 1. Unweighted pairgroup method with arithmetic means tree showing the differences among the 45 mitochondrial haplotypes (Hap.) of Phytophthora cinnamomi identified in this study and their relationship to mating type, location of recovery, host, and isozyme type, as described by Oudemans and Coffey (36). Results represent 6,691 bp from the concatenated data from four mitochondrial genes with flanking spacer sequences ( $\operatorname{cox} 2$, nad9, rps10, and secY) and three mitochondrial spacer regions (trnG-rns, rns-nad3, and nad6-coxl). To ensure that gaps were taken into consideration in the analysis, they were recoded using IUPAC codes prior to running the analysis in PAUP 4.0b10. 
types. For $\mathrm{H} 26$ and $\mathrm{H} 27$ or $\mathrm{H} 39$ and $\mathrm{H} 40$, sequencing the $\sec Y$ locus differentiated haplotypes. Rather than sequencing the entire trnG-rns region, nested sequencing primers that eliminate the first $250 \mathrm{bp}$ will provide the same haplotype results as the entire region. Likewise, designing sequencing primers for the rns-nad3 region that do not include the first $400 \mathrm{bp}$ of the alignment will reduce the amount of sequencing needed but not alter results.

\section{DISCUSSION}

Sequence analysis of the seven mitochondrial loci that were examined revealed a high degree of sequence divergence among isolates of $P$. cinnamomi, with a total of 45 haplotypes identified out of 62 isolates. There were 152 SNPs and 17 indels for a total of 1,062 polymorphic bases in alignments of all isolates. Although some of the differences among haplotypes on a clade were single SNPs or the number of a subrepeated sequence, a greater diversity of SNPs and indels separated the major clades observed in the analysis. Based on the results of the haplotype analysis, there are several examples in the data set where isolates from different geographic locations were the same haplotype (H3, H9, $\mathrm{H} 12, \mathrm{H} 17, \mathrm{H} 26$, and H29), suggesting human involvement in movement of the pathogen. However, to confirm this, it will be necessary to compare these mitochondrial groupings with nuclear genotypic data for the same isolates to evaluate whether there is a correlation between the two genomes.

The most variable regions of the mitochondrial genome of $P$. cinnamomi examined in this study were the intergenic regions

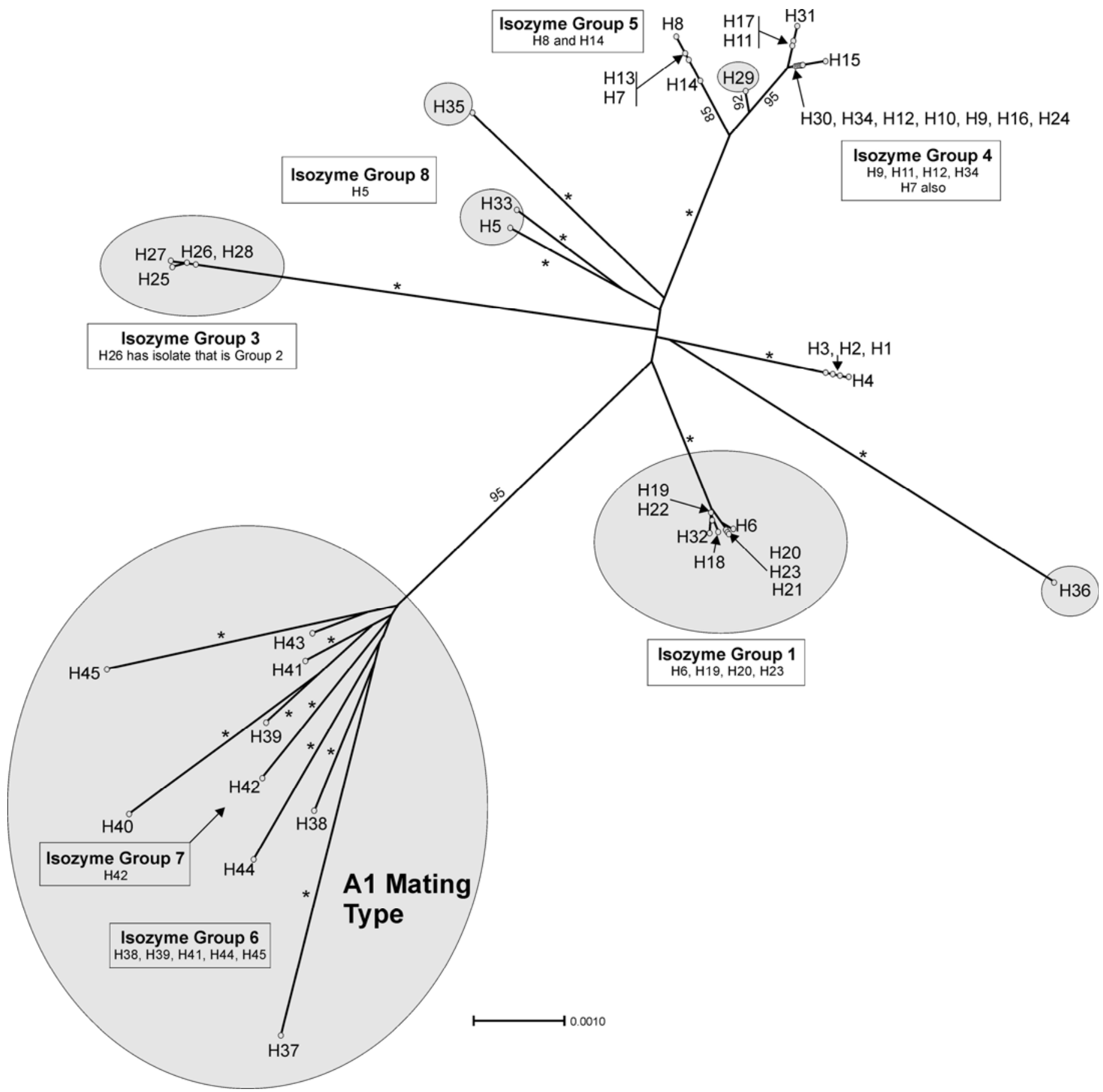

Fig. 2. Mitochondrial haplotype network for Phytophthora cinnamomi calculated by SplitsTree 4.10 (14) using uncorrected $P$ and a neighbor-joining network. Isolates with an A1 mating type are enclosed by the shaded areas and the isozyme grouping of particular isolates from Oudemans and Coffey (36) indicated in boxed text. Bootstrap support for main clades (1,000 replicates) indicated by the smaller numbers on specific branches (if $>99 \%$ indicated by an *). 
trnG-rns, rns-nad3, and nad6-coxl. Although the trnG-rns region had the fewest SNPs $(n=12)$ of these three loci, when combined with a 24-bp subrepeat sequence present in 3 to 12 copies in a putative ORF, this locus differentiated 30 haplotype groups. When looking at the network analysis (Fig. 2), it is this varying number of the 24-bp subrepeat that is responsible for many of the closely spaced haplotype clusters observed on individual branches. For example, $\mathrm{H} 20, \mathrm{H} 21$, and $\mathrm{H} 23$ differ only in the number of these subrepeats, as does $\mathrm{H} 11$ and $\mathrm{H} 17, \mathrm{H} 7$ and $\mathrm{H} 13, \mathrm{H} 26$ and $\mathrm{H} 28$, and $\mathrm{H} 19$ and H22. Interestingly, a change in the number of these repeats was observed in one isolate over time, suggesting that this type of mutation is more prevalent than SNPs or indels. Isolate P2160 was originally isolated in 1971 in South Africa and placed in long-term storage but, in 2002, it was used in a greenhouse pathogenicity trial reisolated from infected tissue and deposited in the World Phytophthora Culture Collection as culture P10140; isolate P2160 had three copies of the 24-bp subrepeat while P10140 had four. In light of this observation, additional studies with multiple isolates are needed to evaluate the long-term stability of this characteristic in haplotype classification.

Schena and Cooke (42) previously examined the trnG-rns region for a range of species and concluded that it was useful for

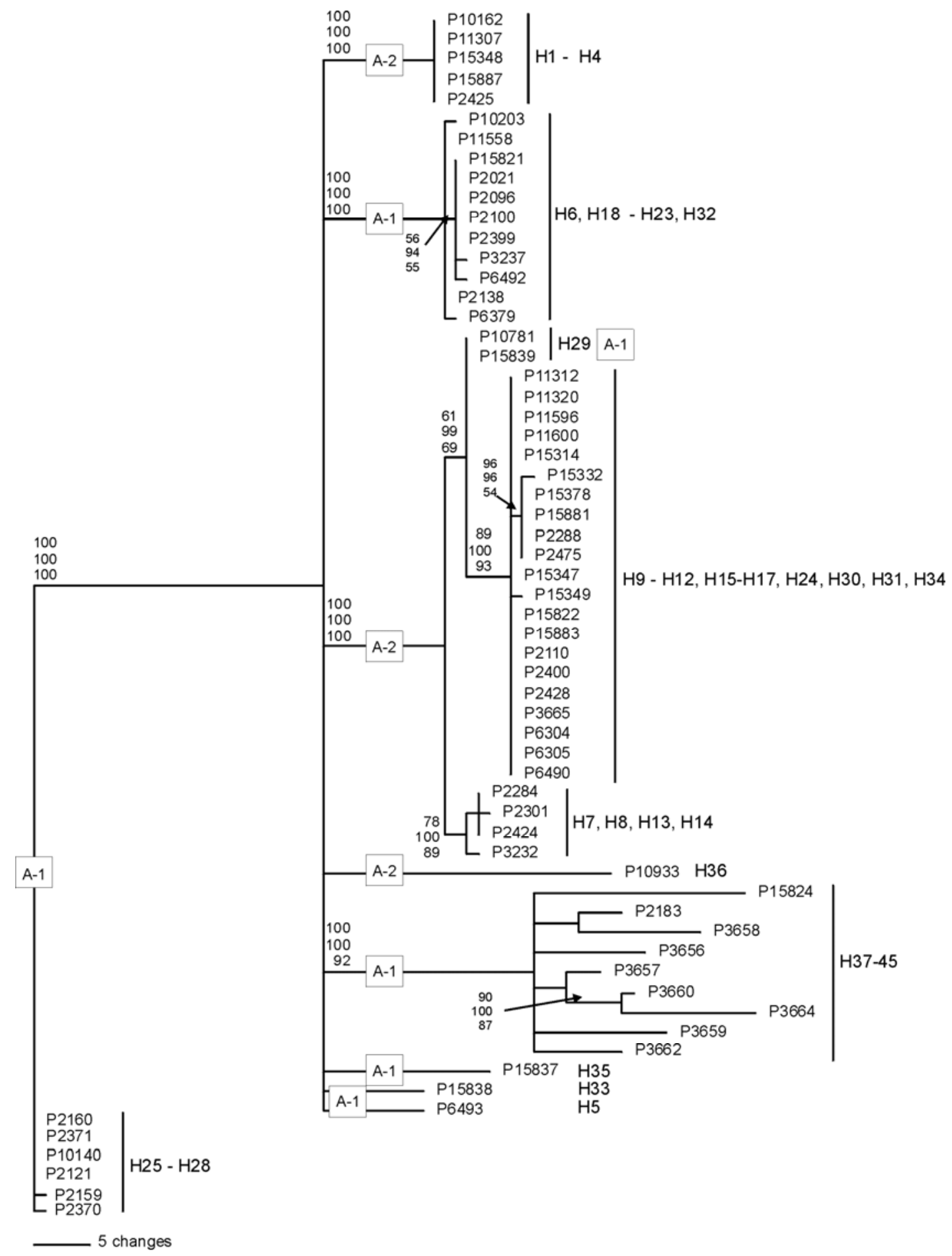

Fig. 3. Maximum parsimony tree for Phytophthora cinnamomi using 6,961 bp from the concatenated data from seven mitochondrial regions (trnG-rns, rns-nad6, nad3-coxl, cox2 and -1, nad9, rps10, and secY) inferred by a heuristic tree search. Numbers at the nodes are the percentage of trees that were supported by bootstrap analysis for maximum parsimony (1,000 replications, bottom number), percent Bayesian posterior probabilities (middle number), and maximum likelihood (100 replications, top number). Matting type is indicated in the box in the clade branch. 
intra- as well as interspecific comparisons; however, they suggested using a forward primer located in the trnY coding region. The tnG-rns amplicon used in the current study spans this same region but generates a larger amplicon because the $\operatorname{trn} G$ is located upstream from $\operatorname{trn} Y$. For $P$. cinnamomi, using the amplicon generated with the $\operatorname{trn} G$ forward primer identified an additional SNP in $\operatorname{trn} G$ (ucc) and a single base indel between the tRNAs that would have been missed if a primer in the $\operatorname{trn} Y$ coding region was used. Intraspecific variation has been observed in this region with P. infestans (1,37), P. nicotianae (20 mitochondrial haplotypes in 51 isolates for this and one other locus; 24), and other Phytophthora spp. as well (F. N. Martin, unpublished); therefore, this larger amplicon should be sequenced in preliminary haplotype analysis to confirm whether additional polymorphisms are present.

The rns-nad3 region had the greatest number of SNPs $(n=67)$ and indels $(n=12)$ of all the loci examined that, combined, generated a total of 24 haplotype groupings. In nearly all cases, the indels were unique sequences with different numbers of subrepeated sequences observed in only two examples; unlike the subrepeat sequence in trnG-rns that varied widely in copy number among isolates, subrepeats in the rns-nad3 intergenic region had a low copy number and were found in only a few isolates. The mutations observed in the nad6-coxl region were primarily SNPs, with only a single indel observed in a few isolates, and allowed for the differentiation of 15 haplotype groups.

Four of the loci examined in this investigation ( $\operatorname{cox} 2, \operatorname{nad} 9$, rps 10, and $\sec Y$ ) contained primarily coding regions with limited amounts of flanking intergenic sequences, yet they were useful for haplotype classification, with 13, 20, 3, and 13 SNPs separating isolates into $10,18,5$, and 11 haplotypes, respectively, when each locus was analyzed individually (indels were rare in these loci, with only one observed in the nad9 and rps10 locus for individual isolates). Portions of the $\operatorname{cox} 2$ - $\operatorname{cox} 1$ gene cluster have been used previously for phylogenetic analysis of Pythium spp. $(25,46)$, Phytophthora spp. $(15,19,28,29,46)$, and a broader analysis of the oomycetes (13) as well as a template for PCRRFLP identification of Phytophthora spp. (30). Due to the polymorphisms in the spacer and coding regions immediately flanking the spacer, this region was also used for the development of a Phytophthora genus- and species-specific diagnostic marker system $(31,45)$. Schena and Cooke (42) also commented on the utility of this spacer region for phylogenetic and taxonomic purposes (their primers $\operatorname{IgCoxF}$ and $\operatorname{IgCoxR}$ are the same as FMPh-8b and FMPh-10b previously reported by Martin et al. [31], with IgCoxF modified with two degenerate bases). However, of the 13 SNPs identified for $P$. cinnamomi with this locus in the current study, 9 of them were located in the cox 2 gene rather than the intergenic region. Inter- as well as intraspecific polymorphisms in the coxl and -2 spacer region have been found with a number of Phytophthora spp. and this region can be very useful for species identification and haplotype analysis (31). Sequence alignments of this region for a wide range of Phytophthora spp. and isolates used in the development of diagnostic markers $(31,45)$ are available for BLAST analysis or they can be downloaded from the diagnostics section of the Phytophthora Database (www.phytophthoradb.org) or Phytophthora ID (www. phytophthora-id.org).

The nad9 locus exhibited the greatest level of intraspecific variation of the four loci spanning coding regions with 20 SNPs, 14 of them located in the intergenic region between the atp 9 and nad9 genes. Schena and Cooke (42) also found this spacer region variable among species but reported amplification from a smaller number of species compared with other mitochondrial regions they amplified (it was not clear if this was due to a lack of amplification of some species or if additional species were not tested). Amplification primers for the nad9 gene used in the current study were initially developed so this gene could be used for phylogenetic analysis of the genus Phytophthora (F. N. Martin, unpublished) and they have successfully amplified templates from virtually all described Phytophthora spp. in a mitochondrial multigene phylogenetic study currently in progress (F. N. Martin, J. Blair, and M. Coffey, unpublished).

The network analysis of the mitochondrial data provided a visual representation of the relationships among the haplotypes and paralleled the results observed with the phylogenetic analysis (MP, ML, and BA). With a few exceptions, the grouping of isolates also agreed with what had been observed for mating type and isozyme grouping. There was one example of a haplotype with an A1 mating type (H29) that grouped well within the A2 mating-type cluster. Although this could be an example of homoplasy, with parallel mutations occurring with this isolate and the A2 isolates, this does not seem likely because it would involve, at a minimum, 11 base changes in five loci. It is more likely that this observation is due to intraspecific crossing that has occurred at some time in the past. Likewise, there were two examples of incongruence when looking at haplotype and isozyme groupings: $\mathrm{H} 26$ grouped with isozyme group 3 isolates but also has an isolate that is in isozyme group 2; and $\mathrm{H} 7$ is in isozyme group 4 but, in the network analysis, grouped with other isolates in isozyme group 5. These isozyme groupings are similar, with each group differing at only one locus; whereas isozyme groups 2 and 3 were homozygous with different electrophoretic types, isozyme group 4 was homozygous and group 5 heterozygous at the same locus, which may also reflect an outcrossing event in the past. Of the eight major clades observed in the network analysis, the greatest diversity in mitochondrial sequences was observed in the clade for A1 isolates from Papua New Guinea (H37 to H39 and H41 to H45), which is consistent with the results of isozyme analysis of Old et al. $(34,35)$ and RAPD and RFLP analysis of Linde et al. (22) and supports the suggestion that the center of origin of the species may be Papua New Guinea. However, to confirm whether this region is the center of origin, additional analysis (such as coalescent analysis) of the mitochondrial data is needed.

The amplification primers used in this experimentation were designed from highly conserved flanking coding regions identified in alignments from data collected from published sequences for $P$. infestans $(1,37), P$. ramorum, and $P$. sojae $(26,27)$ and data from a mitochondrial genome sequencing project for 17 other Phytophthora spp. (F. Martin, unpublished). Alignments also included sequences from Saprolegnia ferax (12) and 14 Pythium spp. (F. Martin, unpublished) and, when possible, the primers were designed from regions conserved for all genera (trnG-rns and rns-nad 3 regions); therefore, it is anticipated that they should amplify these regions for other Phytophthora spp. as long as the gene order is maintained. The trnG-rns region examined in this study is a highly conserved gene order in Phytophthora spp.; however, the rns-nad3 and nad6-coxl gene order are present only in some Phytophthora spp. (F. N. Martin, unpublished). Comparative mitochondrial genomics studies are in progress to clarify gene order differences in relation to phylogenetic grouping in an effort to simplify selection of spacer regions that will be useful for intraspecific comparisons.

In all, 39 of 45 haplotypes (87\%) identified in this experimentation could be classified by sequencing only the trnG-rns and $r n s-n a d 3$ loci, with the remaining six loci differentiated by the nad 9 or $\sec Y$ locus, thereby reducing the sequencing needed for classification. Purified DNA obtained from cultures is required when using the primers designed in this investigation; with the high level of sequence conservation used for primer design, they would be expected to amplify other Phytophthora or Pythium spp. present in environmental samples, thereby complicating haplotype analysis. However, comparison of spacer region sequences from a range of Phytophthora spp. (including those phylogenetically closely related) suggest that it should be possible to 
design species-specific primers that would allow for template amplification directly from infected plant tissue without the need for culturing (F. N. Martin, unpublished). It should also be possible to reduce the time and expense required for haplotype determinations based on sequencing by modifying the primers to amplify templates in the range of $200 \mathrm{bp}$ and using melt curve analysis rather than sequencing. This approach has worked well for haplotype determination with $P$. ramorum, with a single A/T base difference detected (F. N. Martin and L. Radmer, unpublished).

\section{ACKNOWLEDGMENTS}

This work was supported by United States Department of Agriculture Agriculture and Food Research Initiative Plan Biosecurity competitive grant numbers 2007-55605-17835 and 2008-55605-18773. F. N. Martin thanks L. Radmer for her assistance in the collection of the sequence data.

\section{LITERATURE CITED}

1. Avila-Adame, C., Gomez-Alpizar L., Zismann V., Jones K. M., Buell, C. R., and Beagle Ristaino J. 2005. Mitochondrial genome sequences and molecular evolution of the Irish potato famine pathogen, Phytophthora infestans. Curr. Genet. 49:39-46.

2. Bandelt, H. J., Forster, P., and Röhl, A. 1999. Median-joining networks for inferred intraspecific phylogenies. Mol. Biol. Evol. 16:37-48.

3. Blair, J. E., Coffey, M. D., Park, S. Y., Geiser, D. M., and Kang, S. C. 2008. A multi-locus phylogeny for Phytophthora utilizing markers derived from complete genome sequences. Fungal Genet. Biol. 45:266277.

4. Cahill, D. M., Rookes, J. E., Wilson, B. A., Gibson, L., and McDougall, K. L. 2008. Phytophthora cinnamomi and Australia's biodiversity: Impacts, predictions and progress towards control. Aust. J. Bot. 56:279310 .

5. Carter, D. A., Archer, S. A., Buck, K. W., Shaw, D. S., and Shattock, R. C. 1990. Restriction length polymorphisms of mitochondrial DNA of Phytophthora infestans. Mycol. Res. 94:1123-1128.

6. Dobrowolski, M. P., Shearer, B. L., Colquhoun, I. J., O’Brien, P. A., and Hardy, G.-E. St. J. 2008. Selection for decreased sensitivity to phosphite in Phytophthora cinnamomi with prolonged use of fungicide. Plant Pathol. 57:928-936.

7. Dobrowolski, M. P., Tommerup, B. L., Blakeman, H. D., and O'Brien, P. A. 2002. Non-Mendelian inheritance revealed in a genetic analysis of sexual progeny of Phytophthora cinnamomi with microsatellite markers. Fungal Genet. Biol. 35:197-212.

8. Dobrowolski, M. P., Tommerup, B. L., Shearer, B. L., and O'Brien, P. A. 2003. Three clonal lineages of Phytophthora cinnamomi in Australia revealed by microsatellites. Phytopathology 93:695-704.

9. Duan, C. H., Riley, M. B., and Jeffers, S. N. 2008. Characterization of Phytophthora cinnamomi populations from ornamental plants in South Carolina, USA. Arch. Phytopathol. Plant Prot. 41:14-30.

10. Erwin, D. C., and Ribeiro, O. K. 1996. Phytophthora Diseases Worldwide. American Phytopathological Society, St. Paul, MN

11. Förster, H., and Coffey M. D. 1990. Mating behavior of Phytophthora parasitica: Evidence for sexual recombination in oospores using DNA restriction fragment length polymorphisms as genetic markers. Exp. Mycol. 14:351-359.

12. Grayburn, W. S., Hudspeth, D. S. S., Gane, M. K., and Hudspeth, M. E. S. 2004. The mitochondrial genome of Saprolegnia ferax: Organization, gene content, and nucleotide sequence. Mycologia 96:980-987.

13. Hudspeth, D. S. S., Nadler, S. A., and Hudspeth, M. E. S. 2000. A COX2 molecular phylogeny of the peronosporomycetes. Mycologia 92:674-684.

14. Huson, D. H., and Bryant, D. 2006. Applications of phylogenetic networks in evolutionary studies. Mol. Biol. Evol. 23:254-267.

15. Ivors, K., Garbelotto, M., Vries, I., Ruyter, C., Hekkert, B. T. E., Rosenzweig, N., and Bonants, P. 2006. Microsatellite markers identify three lineages of Phytophthora ramorum in US nurseries, yet single lineages in US forest and European nursery populations. Mol. Ecol. 15:1493-1505.

16. Jayasekera, A. U., McComb, J. A., Shearer, B. L., and Hardy, G. 2007. In planta selfing and oospore production of Phytophthora cinnamomi in the presence of Acacia pulchella. Mycol. Res. 111:355-362.

17. Jiang, J., and Erwin, D. C. 1990. Morphology, plasmolosis and tetrazolium bromide stain as a criteria for determining viability of Phytophthora oospores. Mycologia 82:107-113.
18. Jiang, J., Stephenson, L. W., Erwin, D. C., and Leary, J. V. 1989. Nuclear changes in Phytophthora during oospore maturation and germination. Mycol. Res. 92:463-469.

19. Kroon, L. P. N. M., Bakker, F. T., van den Bosch, G. B., Bonnants, P. J., and Flier, W. G. 2004. Phylogenetic analysis of Phytophthora species based on mitochondrial and nuclear DNA sequences. Fungal Genet. Biol. 41:766-782.

20. Librado, P., and Rozas, J. 2009. DnaSP v5: a software for comprehensive analysis of DNA polymorphism data. Bioinformatics 25:1451-1452.

21. Linde, C., Drenth, A., Kemp, G. H. J., Wingfield, M. J., and von Broembsen, S. L. 1997. Population structure of Phytophthora cinnamomi in South Africa. Phytopathology 87:822-827.

22. Linde, C., Drenth, A., and Wingfield, M. J. 1999. Gene and genotype diversity of Phytophthora cinnamomi in South Africa and Australia revealed by DNA polymorphisms. Eur. J. Plant Pathol. 105:667-680.

23. Linde, C, Soo, S. H., and Drenth, A. 2001. Sexual recombination in Phytophthora cinnamomi in vitro and aggressiveness of single-oospore progeny to Eucalyptus. Plant Pathol. 50:97-102.

24. Mammella, M. A., Cacciola, S. O., Martin, F., and Schena, L. 2011. Genetic characterization of Phytophthora nicotianae by the analysis of polymorphic regions of the mitochondrial DNA. Fungal Biol. 115:432442.

25. Martin, F. N. 2000. Phylogenetic relationships among some Pythium species inferred from sequence analysis of the mitochondrially encoded cytochrome oxidase II gene. Mycologia 92:711-727.

26. Martin, F. N. 2008. Mitochondrial haplotype determination in the oomycete plant pathogen Phytophthora ramorum. Curr. Genet. 54:23-34.

27. Martin, F. N., Bensasson, D., Tyler, B. M., and Boore, J. L. 2007. Mitochondrial genome sequences and comparative genomics of Phytophthora ramorum and P. sojae. Curr. Genet. 51:285-296.

28. Martin, F. N., and Tooley, P. W. 2003. Phylogenetic relationships among Phytophthora species inferred from sequence analysis of the mitochondrially-encoded cytochrome oxidase I and II genes. Mycologia 95:269284.

29. Martin, F. N., and Tooley, P. W. 2003. Phylogenetic relationships of Phytophthora ramorum, $P$. nemorosa, and $P$. pseudosyringae, three species recovered from areas in California with sudden oak death. Mycol. Res. 107:1379-1391.

30. Martin, F. N., and Tooley, P. W. 2004. Identification of Phytophthora isolates to species level using restriction fragment length polymorphism analysis of a polymerase chain reaction-amplified region of mitochondrial DNA. Phytopathology 94:983-991.

31. Martin, F. N., Tooley, P. W., and Blomquist, C. 2004. Molecular detection of Phytophthora ramorum, P. nemorosa and P. pseudosyringae associated with sudden oak death in California. Phytopathology 94:621-631.

32. McCarren, K. L., McComb, J. A., Shearer, B. L., and Hardy, G.-E. St. J. 2009. Phosphite impact on the in vitro production and viability of selfed oospores by Phytophthora cinnamomi. For. Pathol. 39:124-132.

33. Milne, I., Wright, F., Rowe, G., Marshal, D. F., Husmeier, D., and McGuire, G. 2004. TOPALi: Software for automatic identification of recombinant sequences within DNA multiple alignments. Bioinformatics 20:1806-1807

34. Old, K. M., Dudzinski, M. J., and Bell, J. C. 1988. Isozyme variability in field populations of Phytophthora cinnamomi in Australia. Aust. J. Bot. 36:355-360.

35. Old, K. M., Moran, G. F., and Bell, J. C. 1984. Isozyme variability among isolates of Phytophthora cinnamomi from Australia and Papua New Guinea. Can. J. Bot. 62:2016-2022.

36. Oudemans, P., and Coffey, M. D. 1991. Isozyme comparisons within and among worldwide sources of three morphologically distinct species of Phytophthora. Mycol. Res. 95:19-30.

37. Paquin, B., Laforest, M.-J., Forget, L., Roewer, I., Wang, Z., Longcore, J., and Lang, B. F. 1997. The fungal mitochondrial genome project: Evolution of fungal mitochondrial genomes and their gene expression. Curr. Genet. 31:380-395.

38. Polzin, T., and Daneschmand, S. V. 2003. On Steiner trees and minimum spanning trees in hypergraphs. Oper. Res. Lett. 31:2-20.

39. Posada, D. 2008. jModelTest: Phylogenetic model averaging. Mol. Biol. Evol. 25:1253-1256.

40. Rands, R. D. 1922. Streepkanker van Kaneel, veroorzaakt door Phytophthora cinnamomi n. sp. Meded. Inst. Plantenziekten 54. (In Dutch)

41. Reeves, R. J., and Jackson, R. M. 1972. Induction of Phytophthora cinnamomi oospores in soil by Trichoderma viride. Trans. Br. Mycol. Soc. 59:156-159.

42. Schena, L., and Cooke, D. E. L. 2006. Assessing the potential of regions of the nuclear and mitochondrial genome to develop a "molecular tool box" for the detection and characterization of Phytophthora species. J. Microbiol. Methods 67:70-85

43. Shearer, B. L., Crane, C. E., and Cochrane, A. 2004. Quantification of the susceptibility of the native flora of the South-West Botanical Province, 
Western Australia, to Phytophthora cinnamomi. Aust. J. Bot. 52:435-443.

44. Stamps, D. J. 1953. Oospore production in paired cultures of Phytophthora species. Trans. Br. Mycol. Soc. 36:255-259.

45. Tooley, P. W., Martin, F. N., Carras, M. M., and Frederick, R. D. 2006. Real-time fluorescent polymerase chain reaction detection of Phytophthora ramorum and Phytophthora pseudosyringae using mitochondrial gene regions. Phytopathology 96:336-345.

46. Villa, N. O., Kageyama, K., Asano, T., and Suga, H. 2006. Phylogenetic relationships of Pythium and Phytophthora species based on ITS rDNA, cytochrome oxidase II and beta-tubulin gene sequences. Mycologia 98:410-422.

47. Whittaker, S. L., Assinder, J. S., and Shaw, D. S. 1994. Inheritance of mitochondrial DNA in Phytophthora infestans. Mycol. Res. 98:569-575.

48. Zentmyer, G. A. 1979. Stimulation of sexual reproduction in the A2 mating type of Phytophthora cinnamomi by a substance in avocado roots. Phytopathology 69:1129-1131. 